\title{
Impact of Covid 19 on Perceived Stress and Social Support during Lockdown
}

\author{
Vidyadayini Shetty $^{1}$, Kinjal Rambhiya ${ }^{2}$ \\ ${ }^{1}$ Associate Professor, Department of Psychology, Nagindas Khandwala College, Mumbai \\ ${ }^{2}$ Student, Department of Psychology, Nagindas Khandwala College, Mumbai. \\ Corresponding author: Vidyadayini Shetty \\ Email: vidhi33@gmail.com
}

\begin{abstract}
Background: Perceived stress is the feelings or thoughts that an individual has about how much stress they are under at a given point in time or over a given time period. Social support represents the amount of support that a person perceives and reports receive it. Social support is a phenomenon that involves interactions of people so that when a person offers social interaction, it has an important role in his health. The present study explores the relationship between perceived stress and social support during Covid 19 lockdown and its relationship with demographic variables such as age, gender, education qualifications and employment status..

Methodology: The Multidimensional Scale of Perceived Social Support (MSPSS) and The Perceived Stress Scale (PSS) was completed by 120 samples - 70 males and 50 females - aged between 18 to 79 years. Descriptive, correlation, and t-test was used for data analysis.

Results: The mean scores of perceived stress and social support was 17.53 and 5.39 respectively. The r value of -.406 obtained for perceived social support with perceived stress was found to be significant. This indicate a moderate levels of perceived stress and perceived social support among the respondents. The $p$ value of 0.04 and 0.006 for perceived stress indicate a significant influence of age and gender. For educational qualifications, a $\mathrm{p}$ value of 0.478 and 0.7436 was obtained for perceived stress and social support. A p value for perceived was 0.001 and for perceived social support $p$ value obtained was 0.00 , for the variable of employment status.

Conclusion: There was a significant negative relationship between perceived stress and perceived social support. Age and gender significantly affected the perceived stress experienced by the respondents during the lockdown period. Education made no difference to perceived stress and perceived social support. Employment status however affected both perceived stress and perceived social support.
\end{abstract}

Keywords: Perceived stress, social support, age, gender, education qualifications, employment status.

(Paper received $-2^{\text {nd }}$ June 2021, Peer review completed $-7^{\text {th }}$ July 2021, Accepted $-14^{\text {th }}$ July 2021)

\section{INTRODUCTION}

Perceived stress is the feelings or thoughts that an individual has about how much stress they are under at a given point in time or over a given time period. Perceived stress incorporates feelings about the uncontrollability and unpredictability of one's life, how often one has to deal with irritating hassles, how much change is occurring in one's life and confidence in one's ability to deal with problems or difficulties. It is not measuring the types or frequencies of stressful events which have happened to a person, but rather how an individual feels about the general stressfulness of their life and their ability to handle such stress [1]. The World Health Organization defines mental health as a state of well-being, that in which the individual knows their capabilities and uses them effectively and productively that will be useful for their respective communities. Social factors, which can play an important role in creating, maintaining, and promoting 
health, have been a major role in incidence, prevalence and persistence of the disease. In this respect, it is very important to pay attention to social factors influencing mental health, and perceived social support is one of those factors. Social support represents the amount of support that a person perceives, and reports receive it. Social support is a phenomenon that involves interactions of people so that when a person offers social interaction, it has an important role in his health. Social support alone is not important, but what is important is the belief in the existence of social support. Social support provides physical and psychological advantages for people faced with stressful physical and psychosocial events and is considered as a factor reducing the psychological distress when faced with stressful events [2].

Due to the spread of Corona virus, Lockdown was imposed in whole country. It was said that lockdown would prove efficient way in order to curb the spread of this infectious diseases. Lockdown brought much panic and stress among all the age group i.e. students were worried about their studies and exams, adults were worried about their jobs and their earnings which was badly affected due to lockdown. All the people were stress and depressed due to this spread of lockdown and spread of Covid-19. But one of the import thing that helped people to cope with an expected situation was the support, strength and encouragement from their families, friends and other peer group. A lockdown of people has been used as an efficient public health measure to fight against the exponential spread of the corona virus disease (Covid-19) and allows the health system to manage the number of patients. The perceived stress resulting from Covid-19 and stress at work and home were also assessed, as were the emotions felt. The results of a study showed that people have experienced a slowing down of time during the lockdown. This time experience was not explained by the levels of perceived stress or anxiety, although these were considerable, but rather by the increase in boredom and sadness felt in the lockdown situation. The conscious experience of time therefore reflected the psychological difficulties experienced during lockdown and was not related to their perceived level of stress or anxiety. Unlike western countries, Indians are thought to be more social and have more social networks, engage in several religious festivals, and get together across the year. This can be attributed to India's diverse culture and traditions. A complete lockdown, therefore, can have a downgrading effect on public psyche. It also leads to long lasting effect on the economy, farming and daily wage earners [3]

Aim

The aim of the current study was to explore the following aspects in reference to the lockdown restrictions employed due to the Covid-19 situation in Mumbai:

- To find the extent of perceived stress and perceived social support among the sample during Covid19 lockdown.

- To explore the gender differences in perceived stress and perceived social support during Covid-19 lockdown.

- To investigate the influence of demographic variables such as age, education qualifications and employment on perceived stress and perceived social support during Covid-19 lockdown

\section{Hypotheses}

- There will be no significant relationship between perceived stress and perceived social support.

- There is no significant difference in the scores of perceived social support and perceived stress among different age groups during Covid-19 lockdown.

- Educational qualifications will not influence the scores of perceived stress and perceived social support experienced during Covid-19 lockdown.

- Employment status will not influence the scores of perceived stress and perceived social support during Covid-19 lockdown.

- Gender will not affect the scores of perceived stress and perceived social support experienced during Covid-19 lockdown.

\section{METHODOLOGY}

\section{Sample}

A total of 120 respondents - 70 males and 50 females - aged between 18 to 79 years, participated in the study. Out of 120 respondents, 30 were employed; 40 unemployed, and 30 self-employed; 25 of them completed 
their SSC/HSC; 64 completed under graduation, and 30 were post graduates. The data was collected in the first week of May, 2020, during the 3rd lockdown restrictions in Mumbai, India.

\section{Procedure}

Snow-ball technique was used to collect the responses using Google forms. The three scales - Perceived Social Support Scale, Perceived Stress Scale and the Self Constructed Scale were prepared and sent to respondents, who were asked to forward it to their friends and relatives. 120 participants answered the questionnaires. The participants also filled the consent form attached to the questionnaire. The participants were asked to fill details about their age, gender, educational qualifications and employment status for further evaluation.

\section{Tools Used}

- Perceived Social support Scale: The Multidimensional Scale of Perceived Social Support (MSPSS) developed by Gregory Zimet is a brief research tool designed to measure perceptions of support from 3 sources: Family, Friends, and a Significant Other. The scale comprised of a total of 12 items, with 4 items for each subscale. Cronbach's coefficient alpha, for significant other, family, friends subscales, were.91, .87 and .85 , respectively. The reliability of the total scale was .88 . These values indicate good internal consistency for the scale as a whole and for three subscales [4].

- Perceived Stress Scale: The Perceived Stress Scale (PSS) is a classic stress assessment instrument developed by Cohen, S. The tool was originally developed in 1983, remains a popular choice for helping us understand how different situations affect our feelings and our perceived stress. The PSS showed adequate reliability and, as predicted, was correlated with life-event scores, depressive and physical symptomology, utilization of health services, and social anxiety [5].

- Self-constructed scale: A socio-demographic questionnaire was designed to collect information concerning age, gender, education level, social status and occupation.

\section{RESULTS \& DISCUSSION}

Table 1: Pearson product-moment correlation coefficients of Perceived Stress Scale (PSS) with Dimensions of Social Support Scale

\begin{tabular}{|c|c|c|c|c|}
\hline & Significant other & Family & Friends & Total \\
\hline PSS & $-.436^{*}$ & $-.422^{*}$ & $-.231^{* *}$ & $-.406^{*}$ \\
\hline
\end{tabular}

The mean scores of perceived stress and social support was 17.53 and 5.39 respectively. This indicate a moderate levels of Perceived stress and social support among the respondents. The first hypothesis investigate if there was significant relationship between the scores of perceived stress and perceived social support. The $r=-.406$ indicate a negative correlation between perceived stress and social support. The $r$ values of the dimensions of perceived social support with perceived stress was: significant other (-.436), family (-.422) and friends (.-231). The data findings indicate a significant negative relationship between perceived stress and the dimensions of perceived social support. In other words, the results obtained indicate that as the perceived social support from significant others, family and friends increases, lower levels of perceived stress experienced among the respondents or vice versa. Similar results were obtained in past studies. The lack of a source of regulation, such as the presence of significant relationships, can exacerbate emotional dysregulation, increasing loneliness and depressive symptoms [6]. Family relationships have taken on an important role, acting as a buffer against stress if they were adequate and supportive, or as a risk factor for depression, if perceived as deficient and inadequate. 
Table 2: Mean, Standard deviation, and $t$ and $F$ values on the score of Perceived Stress Scale and Perceived Social Support in reference to demographic factors

\begin{tabular}{|c|c|c|c|c|c|}
\hline \multicolumn{3}{|c|}{ Perceived Stress Scale } & \multicolumn{3}{|c|}{ Perceived Social Support } \\
\hline \multicolumn{6}{|c|}{ Age } \\
\hline & Mean & SD & $\mathrm{N}$ & Mean & SD \\
\hline $18-20$ & 20.3 & 11.18 & 31 & 5.31 & 1.22 \\
\hline $21-35$ & 18.6 & 5.32 & 29 & 5.25 & 1.38 \\
\hline $36-50$ & 16.0 & 6.2 & 32 & 5.27 & 1.28 \\
\hline $51-79$ & 14.9 & 7.32 & 27 & 5.78 & 0.94 \\
\hline F value & \multicolumn{2}{|c|}{2.86} & \multicolumn{3}{|c|}{1.15} \\
\hline p value & \multicolumn{2}{|c|}{$0.0401^{* *}$} & \multicolumn{2}{|c|}{0.33268} & \\
\hline \multicolumn{6}{|c|}{ Qualifications } \\
\hline SSC \& HSC & 17.8 & 7.21 & 24 & 5.24 & 1.27 \\
\hline Graduation & 18.2 & 9.14 & 63 & 5.45 & 1.2 \\
\hline $\begin{array}{c}\text { Post- } \\
\text { Graduation }\end{array}$ & 66.1 & 6.18 & 32 & 5.25 & 1.27 \\
\hline F value & \multicolumn{2}{|c|}{0.74} & \multicolumn{3}{|c|}{0.30} \\
\hline p value & \multicolumn{2}{|c|}{0.478} & \multicolumn{2}{|c|}{0.7436} & \\
\hline \multicolumn{6}{|c|}{ Employment Status } \\
\hline Employed & 21.2 & 10.01 & 42 & 4.94 & 1.38 \\
\hline Self employed & 14.2 & 5.45 & 48 & 5.73 & 1.09 \\
\hline Unemployed & 17.8 & 6.07 & 29 & 5.48 & 1.02 \\
\hline F value & \multicolumn{2}{|c|}{9.88} & \multicolumn{3}{|c|}{5.08} \\
\hline p value & \multicolumn{2}{|c|}{$0.0001^{* *}$} & \multicolumn{3}{|c|}{$0.0076^{* *}$} \\
\hline \multicolumn{6}{|c|}{ Gender } \\
\hline & $\mathrm{N}$ & Mean & SD & Mean & SD \\
\hline Males & 69 & 15.74 & 6.54 & 5.44 & 1.21 \\
\hline Females & 50 & 20.00 & 9.28 & 5.32 & 1.25 \\
\hline t value & & \\
\hline p value & \multicolumn{3}{|c|}{$\begin{array}{c}-2.78 \\
0.0067^{* *}\end{array}$} & \multicolumn{2}{|c|}{$\begin{array}{c}0.54 \\
0.5931\end{array}$} \\
\hline
\end{tabular}

The second hypothesis investigated the influence of age as a variable on perceived social support and perceived stress of the respondents during the Covid-19 pandemic period. The mean scores point to higher levels of perceived stress among respondents' aged 18 to 20 years, followed by respondents aged between 36-50 years and least perceived stress among the older respondents aged between 51-79 years of age. The $\mathrm{F}$ value of 2.86 indicate a significant difference in perceived stress among the respondents belonging to different age groups. A post-hoc test indicate a significant difference in perceived stress among $18-20$ and 51-59 age groups. The findings also indicated a significant difference in perceived stress among 18-20 years and 36-50 years. However, in respect to perceived social support there was no significant difference in scores among the various age groups. Similar findings were reported by Wilson et al. revealed that at younger ages (1849 years), the positive association between perceived likelihood of contracting COVID-19 and anxiety was significant, but the association was not significant at older ages (50+ years). The study endorse that older adults may be choosing to focus more on the positives of the current moment. Thus, older age may buffer against the negative impact of the COVID-19 pandemic on mental health [7].

The third hypothesis investigates the influence on educational qualifications on perceived social support and perceived stress of the respondents during the Covid-19 pandemic lockdown. People who are educated are more aware of the dynamics of stress they experience. They use their knowledge, skills, and reasoning ability to deal with stress more effectively and efficiently. They are able to able express their problems, which helps to relieve their pent up emotions. The results obtained in the current study indicate no significant difference in perceived stress and perceived social support among respondents belonging to different educational background and consistent with earlier studies. . Past studies have, however, indicated higher levels of 
education were negatively associated with perceived stress and its coefficients. Educational level creates differences between people in terms of access to information and the level of proficiency in benefiting from new knowledge. Accordingly, a lower education placed people at a disadvantaged position for majority of the stressors (i.e., financial stress, worse perceived health status, and psychological distress) and resources (i.e., perceived life control, social support, and social cohesion). Majority of the previous research on the relationships between perceived stress and education level have produced consistent results [8].

The fourth hypothesis investigated the influence of employment on perceived social support and perceived stress of the respondents during the Covid-19 pandemic. Unemployment negatively impacts physical health as reflected in outcomes typically used in medicine, such as a significantly higher likelihood of cardiovascular disease, psychosomatic disorders and subjective physical health [9]. Unemployment has been related to indicators of psychological ill-being such as feelings of depression, anxiety, and decreased life satisfaction [10]. Griep et al. 2014 suggest that (a) insecure employment relates to more psychological complaints than short-term unemployment and secure permanent employment, (b) insecure employment and long-term unemployment relate to more subjective complaints load and poorer health when compared to secure permanent employment, and (c) insecure employment relates to higher life satisfaction than both short- and long-term unemployment [9]. The findings of the present study indicate that employment level significantly influenced the perceived stress and perceived social support scores of respondents, which was not consistent with the Griep et al study. A post hoc test indicate a significant difference in perceived stress among people who are employed and unemployed. The mean scores thus point to higher level of perceived stress among the respondents who are employed, followed by unemployed and least perceived stress among the population who are self-employed. However interesting data was obtained for perceived social support scores. Though there was significant difference in perceived social support scores in the three groups, the results indicate that self-employed perceived significantly higher social support and the least social support was in the employed category. Thus it is presumed that employed respondents experienced higher perceived stress and also little perceived social support. On the other hand self-employed reported lower stress but higher social support. It can be inferred that due to Covid-19 lockdown as commercial establishments were closed and some could not engage in work from home, income was affected. Secondly, due to the unpredictability about the opening up of jobs and other infrastructures, respondents who were employed may have been very anxious about decreasing financial support and retaining their job. Low perceived social support, could be another reason for higher perceived stress among the employed respondents. Those who were self-employed did not have to depend on their bosses or commercial settings, as they were more selfreliant and had opportunity to continue with their businesses.

The fifth hypothesis investigate the influence on gender on perceived stress and perceived social support of the respondents during the Covid-19 pandemic. The $t$ value for perceived stress indicate a significant difference in the mean scores of males and females. In other words, the mean scores indicate, males perceived more stress as compared to female respondents during the lockdown period. However, there was no significant difference in the mean perceived social support scores for males and females. These findings are not consistent with previous studies cited in a research study [11] which revealed that women report higher levels of stress than men. $\mathrm{Ng}$ and Jeffrey report that females are more likely to be stressed than men. The argument advanced by Nolen-Hoeksema that women face a number of burdens in everyday life as a result of social status and roles relative to men and these strains contribute to higher stress perhaps could explain the finding of this study [12]. The findings of the study reveals the techniques used by older adults in dealing with stress can help in developing training practices which can be used by younger adults to deal with their stress. Gender, education and employment factors should be taken into account while forming mental health policies to improve social support systems. Implementing health promoting interventions at school and college level will help to deal with the stressors and help individuals to be self-sufficient in dealing with mental health issues. There are several limitations to the present study. The characteristics of the respondents such as, the limited size of the sample, participant having fair knowledge of English language, possessing technical devices to complete questionnaires using google forms, from urban areas - participated in the study, which cannot be a true representative of the general population. Besides, evaluation of their mental state prior participating in the study was not done. The nature of the study does not identify causeand-effect relationships. 


\section{CONCLUSION}

To sum, the findings of the present study indicate moderate levels of perceived stress and perceived social support experienced by the respondents during the lockdown period. There was a significant negative relationship between perceived stress and perceived social support. Age and gender significantly affected the perceived stress experienced by the respondents during the lockdown period. Employment status however affected both perceived stress and perceived social support.

\section{REFERENCES}

1. Lazarus RS, Folkman S. Stress, Appraisal, and Coping. New York: Springer; 1984.

2. Harandi TF, Taghinasab MM, Nayeri TD. The correlation of social support with mental health: A metaanalysis. Electronic physician 2017;9(9):5212-22.

3. Droit-Volet S, Gil S, Martinelli N, Andant N, Clinchamps M, Parreira L, et al. Time and Covid-19 stress in the lockdown situation: Time free, «Dying» of boredom and sadness. PLoS One 2020;15(8):e0236465.

4. Zimet GD, Dahlem NW, Zimet SG, Farley GK. The multidimensional scale of perceived social support. J Personal Assess 1988;52(1):30-41.

5. Cohen S, Kamarck T, Mermelstein R. A global measure of perceived stress. J Health Soc Behav 1983;24:38596.

6. Mariani R, Renzi A, Di Trani M, Trabucchi G, Danskin K, Tambelli R. The Impact of Coping Strategies and Perceived Family Support on Depressive and Anxious Symptomatology During the Coronavirus Pandemic (COVID-19) Lockdown. Front. Psychiatry 2020;11:587724.

7. Wilson JM, Lee J, Shook NJ. COVID-19 worries and mental health: the moderating effect of age. Aging Ment Health 2021;25(7):1289-96.

8. Feizi A, Aliyari R, Roohafza H. Association of perceived stress with stressful life events, lifestyle and sociodemographic factors: A large-scale community-based study using logistic quantile regression. Computational and Mathematical Methods in Medicine 2012;151865.

9. Griep Y, Kinnunen U, Nätti J, De Cuyper N, Mauno S, Mäkikangas A, De Witte H. The effects of unemployment and perceived job insecurity: a comparison of their association with psychological and somatic complaints, self-rated health and life satisfaction. Int Arch Occup Environ Health 2016;89(1):147-62.

10. Paul KI, Moser K. Unemployment impairs mental health: Meta-analyses. J Vocat Behav 2009;74:264-82.

11. Misigo B. Gender difference in the perceived level of stress and coping strategies among university students in Kenya: A case of public universities Int Acad J Soc Sci Educn 2015;1(4):44-52.

12. Nolen-Hoeksema S. Sex differences in depression. Stanford University Press; 1990.

$* * * * * * * * * * * * * * * * * * * * * * * * * * * * * * * * * * * *$

Acknowledgements - Nil

Conflict of Interest $-\mathrm{Nil}$

Funding - Nil 